\title{
Impact of HIV co-infection on plasma level of cytokines and chemokines of pulmonary tuberculosis patients
}

\author{
Adane Mihret ${ }^{1,2,3^{*}}$, Markos Abebe ${ }^{1}$, Yonas Bekele ${ }^{1}$, Abraham Aseffa ${ }^{1}$, Gerhard Walz| ${ }^{3}$ and Rawleigh Howe
}

\begin{abstract}
Background: The immunologic environment during HIV/M. tuberculosis co-infection is characterized by cytokine and chemokine irregularities that have been shown to increase immune activation, viral replication, and T cell dysfunction.

Methods: We analysed ex vivo plasma samples from $17 \mathrm{HIV}$ negative and $16 \mathrm{HIV}$ pulmonary tuberculosis co infected cases using Luminex assay to see impact of HIV co-infection on plasma level of cytokines and chemokines of pulmonary tuberculosis patients before and after anti Tuberculosis treatment.

Results: The median plasma level of IFN- $\gamma$, IL-4, MCP-3, MIP-1 $\beta$ and IP-10 was significantly different $(P<0.05)$ before and after treatment in HIV negative TB patients but not in HIV positive TB patients. There was no significant difference between HIV positive and HIV negative TB patients $(P>0.05)$ in the plasma level of any of the cytokines or chemokines before treatment and anti TB treatment did not change the level of any of the measured cytokines in HIV positive tuberculosis patients. The ratio of IFN- $\gamma / \mathrm{IL}-10$ and IFN- $\gamma / \mathrm{IL}-4$ showed a significant increase after treatment in HIV negative TB cases but not in HIV positive TB cases which might indicate prolonged impairment of immune response to TB in HIV positive TB patients as compared to HIV negative tuberculosis patients.

Conclusions: HIV positive and HIV negative Tuberculosis patients display similar plasma cytokine and chemokine pattern. However, anti TB treatment significantly improves the Th1 cytokines and level of chemokines but does not restore the immune response in HIV positive individuals.
\end{abstract}

Keywords: Pulmonary tuberculosis, HIV, Cytokines and chemokines

\section{Background}

Tuberculosis (TB) and HIV/AIDS have proven to be a deadly and mutually reinforcing combination. In the absence of anti-retroviral therapy, HIV-infected individuals with latent tuberculosis infection have $5-10 \%$ annual risk of TB disease in contrast to $10 \%$ during life-time in HIV negative individuals [1]. A high risk of TB has been shown in early stages of the HIV disease, even in the presence of normal CD4+ cell counts [2]. The factors contributing to this increased risk are not clear, but may include a compromised response by $\mathrm{CD} 4+\mathrm{T}$ cells, defective

\footnotetext{
* Correspondence: adane_mihret@yahoo.com

${ }^{1}$ Armauer Hansen Research Institute, Addis Ababa, Ethiopia

2Department of Microbiology, Immunology and Parasitology, School of Medicine, College of Health Sciences, Addis Ababa Univrsity, Addis Ababa, Ethiopia

Full list of author information is available at the end of the article
}

innate immunity and/or other elements of the host response against Mycobacterium tuberculosis [3,4].

Understanding what constitutes protective immunity to TB is critical for development of new diagnostics, treatment protocols and identifying vaccine candidates. In order to understand the immune profile of TB disease and infection, a number of parameters need to be considered. These include the HIV status with the majority of diagnostic tests not applicable for HIV positive patients. The cytokine response to TB antigens has been studied extensively but results have varied possibly due to differences in the genetic background of the study population, the type and length of antigen stimulation, the experimental protocol used and the sample type [5-7].

Although the pathogenesis of HIV-tuberculosis coinfection is not well understood, the immunologic environment during $M$. tuberculosis infection is characterized 
by cytokine and chemokine irregularities that are believed to increase $\mathrm{T}$ cell activation, enhance HIV replication and result in a dysfunctional immune response [8-12]. A reduction of Immune activation and HIV viral load after treatment of opportunistic infections other than TB is well recognized. Treatment of TB on the level of cytokines and chemokines among HIV-infected adults with tuberculosis is poorly characterized and effect of TB treatment on viral load and plasma cytokine response have been contradictory where some studies shown an increase pro-inflammatory immune response and decrease HIV viral load in response to TB therapy $[9,13]$ while in other studies not [14-16]. Some studies also shown decrease in immune activation with no effect on viral load [17].

We recently described in a cross-sectional study that anti TB treatment changed the plasma level of cytokines and chemokines in HIV negative TB patients [18]. Here we used the same panel of cytokines to explore the hypothesis that there is a difference between the immune response before and after anti TB treatment in TB patients who are HIV positive compared to those who are HIV negative. We used direct unstimulated plasma samples assuming the sample represents the systemic activation in vivo and reflects the systemic immune response to see the immune response in TB cases who are HIV positive and HIV negative and for monitoring anti $\mathrm{TB}$ treatment outcome in both groups.

\section{Materials and methods}

\section{Study subjects}

We obtained ethical clearance from AHRI/ALERT Ethics Review Committee (P015/10) and National Research Ethics Review Committee (NRERC) (3.10/17/ 10). The participants voluntarily agreed to participate in the study and signed an informed consent form. In this study 33 individuals with active TB disease (17 HIV negative and $16 \mathrm{HIV}$ positive) were recruited and followed from 4 health centers in Addis Ababa, Ethiopia. Diagnosis for TB was made by smear microscopy for AFB. All TB cases were new smear positive pulmonary TB cases and treated for 8 months with 2RHZE/6RE under DOTS and treatment adherence had been assessed by health extension workers. Effective anti TB therapy had been checked and confirmed by negative sputum acid fast bacillus at the end of the treatment.

All sputum samples from TB cases were cultured for mycobacteria. The presence of HIV infection was tested using rapid tests (Stat pack, KHP and Unigold as a tie breaker) as per the national guideline. In Ethiopia all patients attending TB clinics screened for sexually transmitted infections including HIV using a set of simple questions. As soon as HIV is identified in a TB patient, the patient is enrolled to HIV chronic care. The HIV care is delivered at the TB clinic for the duration of TB treatment or the patient may be referred to an HIV Chronic Care/ART clinic. The decision to initiate ART to TB patients is made by a trained clinician based on their CD 4 count. The first priority for HIV-positive TB patients is to initiate TB treatment, followed by cotrimoxazole and ART. HIV positive TB patients with profound immune-suppression (CD4 counts less than 50 cells cells $/ \mathrm{mm} 3$ ) received ART immediately within the first 2 weeks of initiating TB treatment. Otherwise, timing of ART initiation is up to clinical judgment based on other signs of immunodeficiency indicating progression of HIV disease [19].

\section{Multiplex analysis}

Blood sample was collected with heparin tubes twice at recruitment and after completion of anti TB treatment. Plasma was separated by centrifugation at $1000 \mathrm{rpm}$ for 5 min and stored at -20 until the multiplex analysis was performed.

We used a 17plex kit (Epidermal Growth Factor (EGF), FRACTALKINE, Granulocyte Macrophage Colony Stimulating Factor (GM CSF), IFN- $\gamma$, IL-1, IL 10, IL-12, IL-17, IL-4, IL-7, IL-9, IFN- $\gamma$ inducible protein (IP-10 /CXCL10), Macrophage Chemo-attractant Protein 1 (MCP- 1/ CXCL), MCP-3, Monocyte Inflammatory Protein 1 beta (MIP-1 $\beta$ ), TNF and VEGF) from Millipore, Germany and multicytokine analysis was done using Luminex (Millipore, Germany) technology. The assays were performed according to the supplier's instructions. Briefly, following pre wetting of plates, $50 \mu \mathrm{l}$ of precombined beads from all the 17 individual cytokines or chemokines was added and washed twice after $1 \mathrm{hr}$ incubation at room temperature. Plasma samples $(25 \mu \mathrm{l})$ were diluted 1:1 with the kit serum matrix and added to the plate. The plate was shaken for $30 \mathrm{sec}$ at $1000 \mathrm{RPM}$ and then incubated for $1 \mathrm{hr}$ on plate shaker (300 RPM) at room temperature. The plates were washed twice and $25 \mathrm{ul}$ of detection antibody was added per well and incubated for $1 \mathrm{hr}$ again on a plate shaker. Fifty micro liter of streptavidin-PE conjugate was added per well and incubated for $30 \mathrm{~min}$ at room temperature. Finally, the plate was washed 3 times and $150 \mu \mathrm{l}$ of sheath fluid was added to each well and then the plate was read by Luminex machine (Millipore, Germany). Data was analysed using Luminex 100 IS software version 2.3.182. The Human Cytokine Quality Controls 1 and 2 and Assay buffer included in the kit were used as low and high concentration quality controls and background (Blank) respectively. Six non zero standard points ranging from $3.2 \mathrm{pg} / \mathrm{ml}$ to $10000 \mathrm{pg} / \mathrm{ml}$ were assayed in duplicate to generate standard curve and the correlation coefficient $\left(R^{2}\right)$ was calculated in each experiment to see the linearity of the standard curve. 


\section{Statistical analysis}

The data were analyzed using Graph pad prism software, version 4.0. Data were log transformed to normalize their distribution. Nonparametric Mann-Whitney U tests were performed to check for the significance of the observed differences in each parameter in HIV positive and HIV negative TB cases.

\section{Results}

We enrolled 33 subjects with active tuberculosis of which 16 were HIV positive and all were ART naïve with a mean CD4 count of $310 \pm 23.9$ cells $/ \mu \mathrm{L}$. The mean age of HIV positive TB patients and HIV negative TB patients was $29.6 \pm 1.3$ and $31.8 \pm 1.2$ respectively and $48.5 \%$ of the participants were females.

\section{Effect of HIV on plasma level of cytokines and chemokines on TB patients}

We found that the median plasma level of the cytokines and chemokines measured was not affected by HIV status although the HIV positive TB patients have a slightly higher level of most of the cytokines and chemokines (Figure 1).
Impact of anti TB treatment on plasma level of cytokines and chemokines

We also measured the plasma level of the cytokines and chemokines after 8 month of effective anti-TB therapy to see if any of these cytokines and chemokines showed a difference among HIV positive and HIV negative TB patients as a result of treatment. We found that the median level of IFN- $\gamma$, IL-4, IP-10, MCP-3 and MIP-1 $\beta$ were statistically different $(\mathrm{p}<0.05)$ after treatment in HIV negative TB patients but not in HIV positive TB patients $(\mathrm{p}>0.05)$ (Figure 2). The median plasma level of IL-4 and IP-10 was significantly decreased whereas the level of IFN- $\gamma$, MCP-3 and MIP-1 $\beta$ significantly increased after treatment in HIV negative TB patients.

We also analysed the ratio of Th1 (IFN- $\gamma$ and IL-12 (p40)) and Th2 (IL-4 and IL-10) cytokines before and after treatment. None of the ratios of IFN- $\gamma / \mathrm{IL}-4$, IFN$\gamma / \mathrm{IL}-10, \mathrm{IL}-12(\mathrm{p} 40) / \mathrm{IL}-4$ and IL-12(p40)/IL-10 were significantly different between TB patients who are HIV positive or HIV negative (Figure 2). Whereas the plasma concentration of IFN- $\gamma / \mathrm{IL}-4$ and IFN- $\gamma / / \mathrm{IL}-10$ was significantly higher $(\mathrm{p}<0.05)$ in HIV negative TB patients than HIV positive TB patients after treatment (Figure 3).
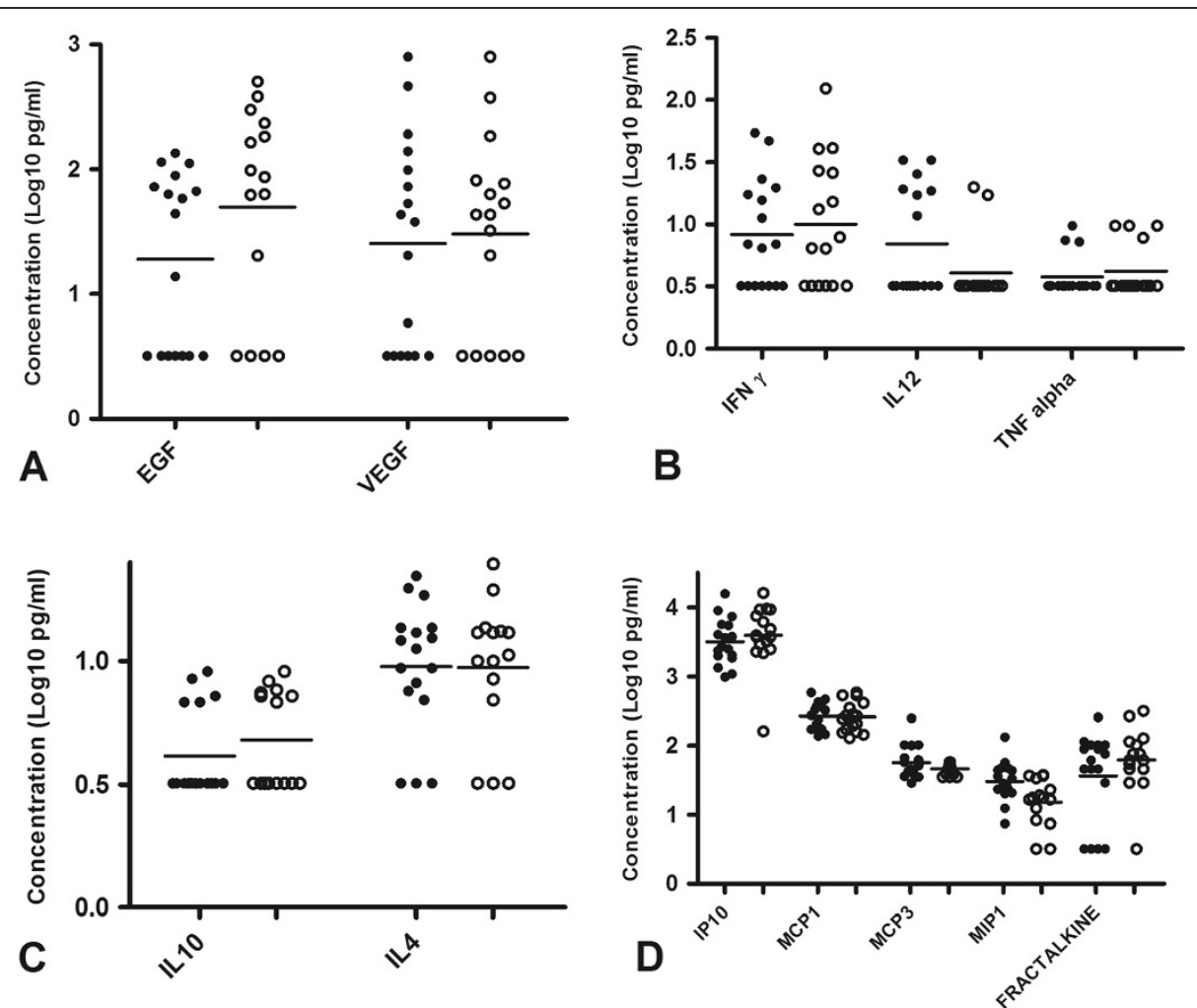

Figure 1 Plasma cytokine and chemokine level in HIV negative and HIV positive TB cases. Unstimulated plasma samples from HIV positive TB cases $(n=16)$ and HIV negative TB cases $(n=17)$ were assessed by multiplex cytokine analysis. A) Growth factors (EGF and VEGF) B) Pro-inflammatory cytokines (IFN-y, IL 12(p40), and TNF) C) Anti-inflammatory cytokines (IL-10 and IL-4) and D) Chemokines (IP-10, MCP-1, MCP-3, MIP-1 $\beta$ and fractalkine). Horizontal lines indicate median of HIV negative (filled circles) and HIV positive TB cases (open circles). Data were analysed using nonparametric Mann-Whitney test with $\mathrm{p}$-values indicating significant differences after transformation of data to Log10 values. 


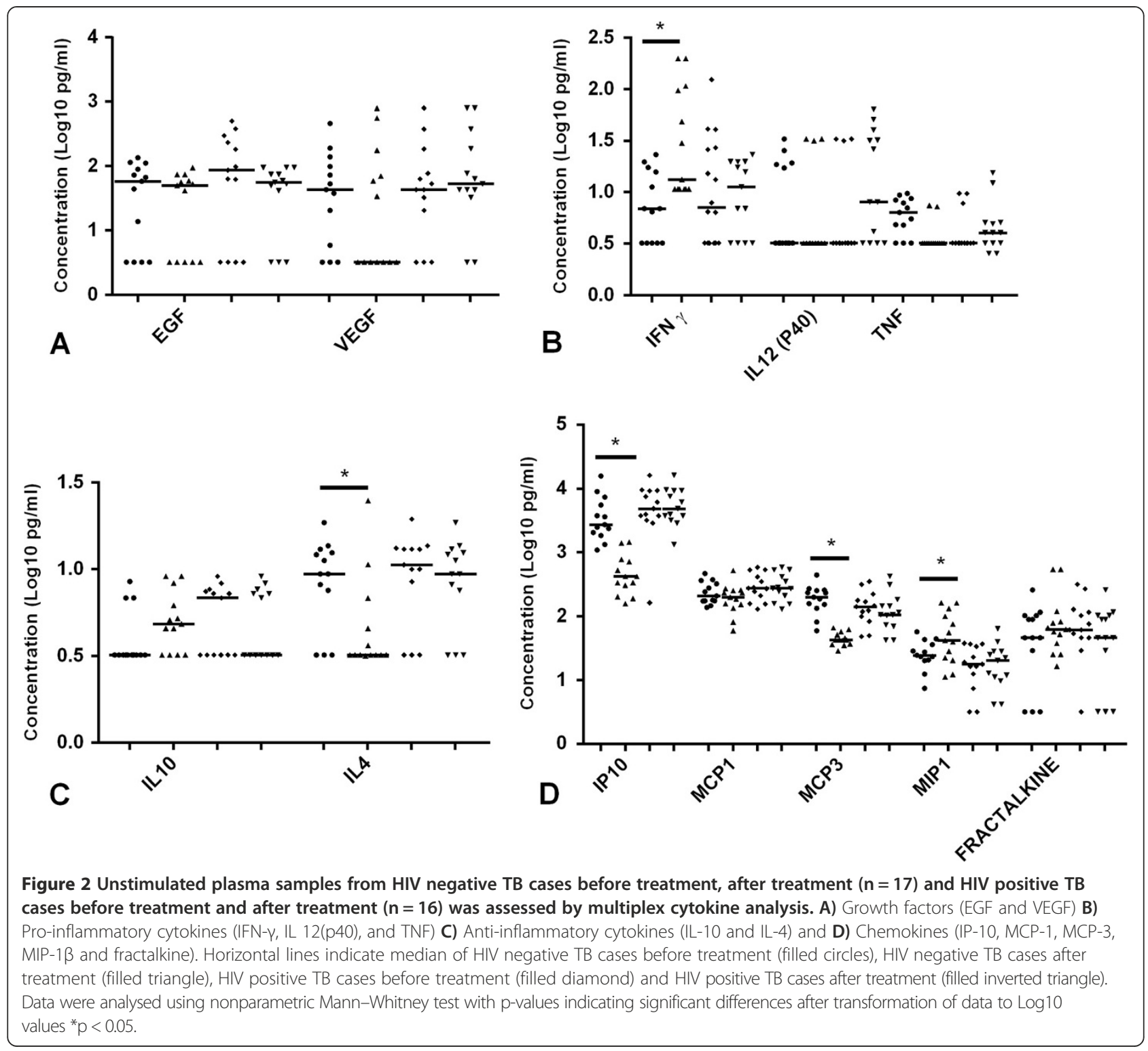

\section{Discussion}

This study identifies differences in cytokine levels in TB patients with and without HIV and before and after anti TB treatment that may potentially plays a role in the protection or development of TB. We measured the plasma level of pro-inflammatory cytokines (IFN- $\gamma$, IL 12(p40), and TNF), Anti-inflammatory cytokines (IL-10 and IL-4), Growth factors (EGF and VEGF) and Chemokines (IP-10, MCP-1, MCP-3, MIP-1 $\beta$ and fractalkine). The main findings of this study were: (a) HIV coinfection does not seem to affect the median plasma level of any of the cytokine/chemokine in TB patients and there was no statistically significant difference between HIV positive and HIV negative TB patients; (b) The plasma level of IFN- $\gamma$, IL-4, IP-10, MCP-3 and MIP$1 \beta$ significantly changed after completion of anti-TB treatment in HIV negative TB patients whereas in HIV positive TB patients none of the cytokines and chemokines showed significant difference after anti TB treatment; (c) The ratio of IFN- $\gamma / \mathrm{IL}-4$ and IFN- $\gamma / \mathrm{IL}-10$ were statistically different $(\mathrm{p}<0.05)$ in HIV negative TB patients after treatment; and (d) The ratio of Th1/Th2 cytokines in HIV positive TB patients was not changed after treatment and it was significantly lower than HIV negative tuberculosis cases after anti TB treatment.

At baseline, TB + HIV + individuals displayed a similar plasma cytokine level when compared to TB + HIV- individuals. This could be due to both tuberculosis and HIV infection inducing immune activation in a similar pattern or due to the nature of HIV positive participants in this study where majority of them were at less advanced HIV infection stage as confirmed by their CD4 


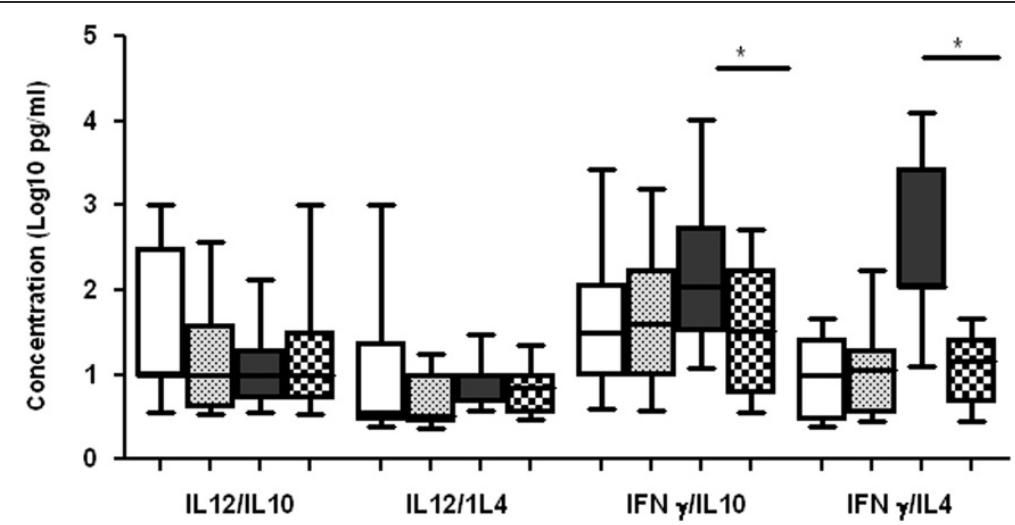

Figure 3 Th1/Th2 ratios of cytokines of TB cases after anti TB treatment. Box plots are shown with the horizontal line indicating median levels of TB cases and the lower and upper edge of each box indicate the 25th and 75th percentiles, respectively. Ratio of Th1/Th2 cytokines (IL-12/IL-10, IL-12/IL-4, IFN- $\gamma /$ IL-10 and IFN- $\gamma /$ IL-4) of TB patients who are HIV negative before treatment (white bars), TB patients who are HIV positive before treatment (Dotted bars), TB patients who are HIV negative after treatment (grey bars) and TB patients who are HIV positive after treatment negative (crossed bars). Data were analysed using nonparametric Mann-Whitney test with $p$-values indicating significant differences after transformation of data to Log10 values.

count. In our previous study we have shown that TB patients have a similar plasma cytokine and chemokine level with healthy household contacts after completion of anti TB treatment [18]. Different studies have shown that both tuberculosis and HIV infection can inhibit $\mathrm{T}$ cell effector functions, such as production of IFN- $\gamma$ and interleukin-2, and co-infection is associated with more profound suppression of type-1 cytokine responses [10,20,21].

Longitudinal assessment of the plasma cytokine profile showed that out of the 17 cytokines measured, the plasma level of IFN- $\gamma$, IL-4, IP-10, MCP-3 and MIP-1 $\beta$ significantly changed after completion of anti-TB treatment in HIV negative TB patients whereas in HIV positive TB patients none of the cytokines and chemokines showed significant difference after anti TB treatment. The median plasma level of IL-4 and IP-10 was significantly decreased whereas the level of IFN- $\gamma$, MCP-3 and MIP-1 $\beta$ significantly increased after treatment. Because IFN- $\gamma$ is a pro-inflammatory and IL- 4 is an antiinflammatory cytokine, it is reasonable to suggest that the increased concentration of IFN- $\gamma$ and the reduced concentration of IL-4 might be due to the clearance of the actively multiplying bacteria and resolution of the disease following effective anti-tuberculosis treatment. The decrement of IP-10 and increment of MCP-1 and MIP-1 $\beta$ after treatment has been also reported by other studies [22-24]. IP-10 is involved in trafficking monocytes and activated Th1 cells to inflamed foci and the reduction of this chemokine could be due to resolution of inflammation as a result of anti TB treatment. The increased concentration of MCP-1 and MIP-1b is significant because of the key role these two cytokines play in the control of infection by attracting cells to the granuloma. In TB + HIV + patients the level of all of the cytokines and chemokines measured was not changed and this could be due to the ongoing inflammatory immune response.

The ratio of Th1/Th2 in HIV + and HIV- TB patients showed no difference before treatment whereas the ratio of IFN- $\gamma / \mathrm{IL}-4$ and IFN- $\gamma / \mathrm{IL}-10$ showed a significant difference before and after treatment in HIV negative TB cases where as none of the Th1/Th2 cytokines ratio showed a significant difference after treatment in HIV positive TB cases. The ratio of Th1/Th2 cytokines in HIV positive TB patients was lower than HIV negative TB cases. The higher level of IFN- $\gamma / \mathrm{IL}-4$ and IFN- $\gamma / \mathrm{IL}-10$ in HIV negative TB cases is expected where a Th1 type immune response dominates after clearance of the actively multiplying bacteria and resolution of the disease by the anti-tuberculosis treatment. In HIV positive TB patients anti TB treatment did not change the level of circulatory cytokines. It remains unclear whether tuberculosis treatment has a significant impact on other markers of HIV disease progression, such as $\mathrm{T}$ cell counts and changes in $\mathrm{T}$ cell subsets. Some studies reported that tuberculosis treatment increased CD4 T cell count among persons with HIV-tuberculosis co infection $[13,25]$ whereas others indicated anti TB treatment didn't show any change in absolute CD4 $\mathrm{T}$ cell count or restoration of the naive $T$ cell population [26].

The lower ratio of IFN- $\gamma / \mathrm{IL}-4$ and IFN- $\gamma / \mathrm{IL}-10$ after anti TB treatment in HIV positive patients could be due to immune exhaustion by HIV co-infection with a possibility of future development of TB in HIV-infected individuals through down-regulation of IFN- $\gamma$ and IL-12 and up-regulation of IL-4 and IL-10. The higher level of IL10 and IL-4 in co-infected TB patients might be due to the immune exhaustion by HIV where the HIV-specific 
CD4 T cell response is inhibited through upregulation of IL-4 and IL-10. Previous studies suggested that the level of IL-10 is elevated in chronic HIV disease [27]. Moreover, lower IL-12 production by PBMC in response to TB antigens and lower levels of both Th1 and Th2 cytokines by PBMC correlate with increased susceptibility and development of TB in HIV-infected individuals and may be responsible for their increased susceptibility [28]. Moreover, a reduced ratio of IFN- $\gamma / \mathrm{IL}-4$ might indicate increased disease severity in HIV positive TB patients as lower ratio of IFN- $\gamma / \mathrm{IL}-10$ has been found to relate with increased disease severity in pulmonary and extrapulmonary TB [29].

The findings in this study however need further detailed analysis including (i) larger sample size, (ii) viral load and (iii) CD 4 count for HIV negative TB cases and after anti TB treatment for both groups to validate and confirm that the observed dysregulation of cytokine and chemokine production are indeed associated with increased susceptibility.

\section{Conclusions}

HIV positive and HIV negative Tuberculosis patients display similar plasma cytokine and chemokine pattern. However, anti TB treatment significantly improves the Th1 cytokines and level of chemokines but does not restore the immune response in HIV positive individuals. This finding may suggest that in dually infected subjects, the HIV-related changes dominate the overall immunological picture and leads to dysregulation of cytokine and chemokine production.

\section{Competing interests}

No conflict of interest.

\section{Authors' contributions}

AM involved in study design, data collection and analysis, data interpretation and drafted the manuscript. MA involved in analysis and write-up. YB involved in sample collection and laboratory work. AA, GW and RH were involved in study initiation, interpretation and write up of the manuscript. All authors read and approved the final manuscript.

\section{Acknowledgements}

We acknowledge the invaluable contribution to this study made by Bamlak Tessema, Sr. Semegne Tesfaye, Sr. Etsegenet Aseffa, Sr. Tigest Gezmu and Sr. Sefina Juhar in recruiting and following study participants. This research is supported by the Bill and Melinda Gates Foundation (BMGF) through Grand Challenges in Global Health (GCGH), grant no. 37772 and the European and Developing Countries Clinical Trial Partnership (EDCTP) through the African European Tuberculosis Consortium (AETBC), grant no.IP_2009_32040.

\section{Author details}

${ }^{1}$ Armauer Hansen Research Institute, Addis Ababa, Ethiopia. ${ }^{2}$ Department of Microbiology, Immunology and Parasitology, School of Medicine, College of Health Sciences, Addis Ababa Univrsity, Addis Ababa, Ethiopia. ${ }^{3}$ DST/NRF Centre of Excellence for Biomedical Tuberculosis Research and MRC Centre for Molecular and Cellular Biology, Division of Molecular Biology and Human Genetics, Faculty of Medicine and Health Sciences, Stellenbosch University, PO Box 19063, Francie van Zijl Drive, Tygerberg 7505, South Africa.
Received: 15 November 2013 Accepted: 26 February 2014

Published: 4 March 2014

\section{References}

1. Rook GA, Dheda K, Zumla A: Immune responses to tuberculosis in developing countries: implications for new vaccines. Nat Rev Immunol 2005, 5:661-667.

2. Sonnenberg P, Glynn JR, Fielding K, Murray J, Godfrey-Faussett P, Shearer S: How soon after infection with HIV does the risk of tuberculosis start to increase? A retrospective cohort study in South African gold miners. $J$ Infect Dis 2005, 191:150-158.

3. North RJ, Jung YJ: Immunity to tuberculosis. Annu Rev Immunol 2004, 22:599-623.

4. van Crevel R, Ottenhoff TH, van der Meer JW: Innate immunity to Mycobacterium tuberculosis. Clin Microbiol Rev 2002, 15:294-309.

5. Sallusto F, Lenig D, Forster R, Lipp M, Lanzavecchia A: Two subsets of memory T lymphocytes with distinct homing potentials and effector functions. Nature 1999, 401:708-712.

6. Martinez V, Carcelain G, Badell E, Jouan M, Mauger I, Sellier P, Truffot C, Bricaire F, Arend SM, Ottenhoff T, Autran B, Gicquel B: T-cell and serological responses to Erp, an exported Mycobacterium tuberculosis protein, in tuberculosis patients and healthy individuals. BMC Infect Dis 2007, 7:83.

7. Demissie A, Leyten EM, Abebe M, Wassie L, Aseffa A, Abate G, Fletcher H, Owiafe P, Hill PC, Brookes R, Rook G, Zumla A, Arend SM, Klein M, Ottenhoff $T H$, Andersen P, Doherty TM: Recognition of stage-specific mycobacterial antigens differentiates between acute and latent infections with Mycobacterium tuberculosis. Clin Vaccine Immunol 2006, 13:179-186.

8. Toossi Z, Johnson JL, Kanost RA, Wu M, Luzze H, Peters P, Okwera A, Joloba M, Mugyenyi P, Mugerwa RD, Aung H, Ellner JJ, Hirsch CS: Increased replication of HIV-1 at sites of Mycobacterium tuberculosis infection: potential mechanisms of viral activation. J Acquir Immune Defic Syndr 2001, 28:1-8.

9. Goletti D, Weissman D, Jackson RW, Graham NM, Vlahov D, Klein RS, Munsiff SS, Ortona L, Cauda R, Fauci AS: Effect of Mycobacterium tuberculosis on HIV replication. Role of immune activation. J Immunol 1996, 157:1271-1278.

10. Toossi Z, Mayanja-Kizza H, Hirsch CS, Edmonds KL, Spahlinger T, Hom DL, Aung H, Mugyenyi P, Ellner JJ, Whalen CW: Impact of tuberculosis (TB) on HIV-1 activity in dually infected patients. Clin Exp Immunol 2001, 123:233-238.

11. Hertoghe T, Wajja A, Ntambi L, Okwera A, Aziz MA, Hirsch C, Johnson J, Toossi Z, Mugerwa R, Mugyenyi P, Colebunders R, Ellner J, Vanham G: $T$ cell activation, apoptosis and cytokine dysregulation in the (co) pathogenesis of HIV and pulmonary tuberculosis (TB). Clin Exp Immunol 2000, 122:350-357.

12. Vanham G, Edmonds K, Qing L, Hom D, Toossi Z, Jones B, Daley CL, Huebner B, Kestens L, Gigase P, Ellner Jj: Generalized immune activation in pulmonary tuberculosis: co-activation with HIV infection. Clin Exp Immunol 1996, 103:30-34.

13. Dean GL, Edwards SG, Ives NJ, Matthews G, Fox EF, Navaratne L, Fisher M, Taylor GP, Miller R, Taylor CB, de Ruiter A, Pozniak AL: Treatment of tuberculosis in HIV-infected persons in the era of highly active antiretroviral therapy. AIDS 2002, 16:75-83.

14. Kalou M, Sassan-Morokro M, Abouya L, Bile C, Maurice C, Maran M, Tossou O, Roels T, Greenberg AE, Wiktor SZ, Nkengasong JN: Changes in HIV RNA viral load, CD4+ T-cell counts, and levels of immune activation markers associated with anti-tuberculosis therapy and cotrimoxazole prophylaxis among HIV-infected tuberculosis patients in Abidjan, Cote d'Ivoire. J Med Virol 2005, 75:202-208.

15. Morris L, Martin DJ, Bredell H, Nyoka SN, Sacks L, Pendle S, Page-Shipp L, Karp CL, Sterling TR, Quinn TC, Chaisson RE: Human immunodeficiency virus-1 RNA levels and CD4 lymphocyte counts, during treatment for active tuberculosis, in South African patients. J Infect Dis 2003, 187:1967-1971.

16. Lawn SD, Shattock RJ, Acheampong JW, Lal RB, Folks TM, Griffin GE, Butera ST: Sustained plasma TNF-alpha and HIV-1 load despite resolution of other parameters of immune activation during treatment of tuberculosis in Africans. AIDS 1999, 13:2231-2237.

17. Mahan CS, Walusimbi M, Johnson DF, Lancioni C, Charlebois E, Baseke J, Chervenak KA, Mugerwa RD, Havlir DV, Mayanja-Kizza H, Whalen CC, Boom WH: Tuberculosis treatment in HIV infected Ugandans with CD4 
counts $>350$ cells $/ \mathrm{mm}$ reduces immune activation with no effect on HIV load or CD4 count. PLoS One 2010, 5:e9138.

18. Mihret A, Bekele Y, Bobosha K, Kidd M, Aseffa A, Howe R, Walzl G: Plasma cytokines and chemokines differentiate between active disease and non-active tuberculosis infection. J Infect 2012, 66:357-365.

19. Federal democratic republic of Ethiopia: Guidelines for Clinical and Programmatic Management Of TB, TB/HIV and Leprosy. 5th edition. City: Ministry of health; 2013.

20. Zhang M, Gong J, lyer DV, Jones BE, Modlin RL, Barnes PF: T cell cytokine responses in persons with tuberculosis and human immunodeficiency virus infection. J Clin Invest 1994, 94:2435-2442.

21. Sutherland R, Yang H, Scriba TJ, Ondondo B, Robinson N, Conlon C, Suttill A, McShane H, Fidler S, McMichael A, Dorrell L: Impaired IFN-gammasecreting capacity in mycobacterial antigen-specific CD4 T cells during chronic HIV-1 infection despite long-term HAART. AIDS 2006, 20:821-829.

22. Azzurri A, Sow OY, Amedei A, Bah B, Diallo S, Peri G, Benagiano M, D'Elios MM, Mantovani A, Del Prete G: IFN-gamma-inducible protein 10 and pentraxin 3 plasma levels are tools for monitoring inflammation and disease activity in Mycobacterium tuberculosis infection. Microbes Infect 2005, 7:1-8.

23. Siawaya J, Beyers N, van Helden P,GW: Differential cytokine secretion and early treatment response in patients with pulmonary tuberculosis. I Infect Dis 2009, 156:69-77.

24. Riou C, Perez Peixoto B, Roberts L, Ronacher K, Walzl G, Manca C, Rustomjee R, Mthiyane T, Fallows D, Gray CM, Kaplan G: Effect of standard tuberculosis treatment on plasma cytokine levels in patients with active pulmonary tuberculosis. PLoS One 2012, 7:e36886.

25. Bohler T, Walcher J, Holzl-Wenig G, Geiss M, Buchholz B, Linde R, Debatin KM: Early effects of antiretroviral combination therapy on activation, apoptosis and regeneration of T cells in HIV-1-infected children and adolescents. AIDS 1999, 13:779-789.

26. Lancioni CL, Mahan CS, Johnson DF, Walusimbi M, Chervenak KA, Nalukwago S, Charlebois E, Havlir D, Mayanja-Kizza H, Whalen CC, Boom WH: Effects of antiretroviral therapy on immune function of HIV-infected adults with pulmonary tuberculosis and CD4+ >350 cells $/ \mathrm{mm} 3$. J Infect Dis 2011, 203:992-1001.

27. Kwon DS, Angin M, Hongo T, Law KM, Johnson J, Porichis F, Hart MG, Pavlik DF, Tighe DP, Kavanagh DG, Streeck H, Addo MM, Kaufmann DE: CD4+ CD25+ regulatory T cells impair HIV-1-specific CD4 T cell responses by upregulating interleukin-10 production in monocytes. J Virol 2012, 86:6586-6594

28. Bordon J, Plankey MW, Young M, Greenblatt RM, Villacres MC, French AL, Zhang J, Brock G, Appana S, Herold B, Durkin H, Golub JE, Fernandez-Botran R: Lower levels of interleukin-12 precede the development of tuberculosis among HIV-infected women. Cytokine 2011, 56:325-331.

29. Jamil B, Shahid F, Hasan Z, Nasir N, Razzaki T, Dawood G, Hussain R: Interferon gamma/LL10 ratio defines the disease severity in pulmonary and extra pulmonary tuberculosis. Tuberculosis (Edinb) 2007, 87:279-287.

doi:10.1186/1471-2334-14-125

Cite this article as: Mihret et al:: Impact of HIV co-infection on plasma level of cytokines and chemokines of pulmonary tuberculosis patients. BMC Infectious Diseases 2014 14:125.

\section{Submit your next manuscript to BioMed Central and take full advantage of:}

- Convenient online submission

- Thorough peer review

- No space constraints or color figure charges

- Immediate publication on acceptance

- Inclusion in PubMed, CAS, Scopus and Google Scholar

- Research which is freely available for redistribution 